\title{
Comparing the Effectiveness of Electroacupuncture with Different Grades of Knee Osteoarthritis: A Prospective Study
}

\author{
Li Qi Yonggang Tang Yong You Fengling Qin Lijuan Zhai Hongxia Peng \\ Rongrong Nie
}

Department of Neurological Rehabilitation, 181st Central Hospital of The Chinese People's Liberation Army, Guilin, Guangxi, China

\section{Key Words}

Electroacupuncture $\cdot$ Osteoarthritis $•$ Clinical effectiveness $\bullet$ VAS $・$ WOMAC

\begin{abstract}
Background: The electroacupuncture (EA) with different number of points significantly affected its efficacy on knee osteoarthritis (KOA), and the severity of KOA also influenced its response to treatments. Hence, we prospectively compared the clinical efficacy of EA on KOA with different severities. Methods: A total of $132 \mathrm{KOA}$ patients recruited from 181st Central Hospital of The Chinese People's Liberation Army between March 2014 and March 2015 were classified into $4 \mathrm{KOA}$ stages according to Kellgren Lawrence grading scale. They were allocated into three treatment groups, including two-point group, four-point group and sixpoint group. Patients in the six-point group received treatment at six-points including ST34, SP10, SP9, ST36, ST35 and EX-LE4. Patients in the four-point group received treatment at ST34, SP10, ST35 and EX-LE5, while patients in the two-point group received treatment at ST35 and EX-LE4. Visual Analog Scale (VAS), McMaster Universities Osteoarthritis Index (WOMAC) and self-assessment questionnaire of patients were assessed after treatment. Results: Three kinds of EA treatments all have significant clinical effects on KOA patients with down-regulated scores of VAS and WOMAC. Regarding post-treatment efficacy, the six point group exhibited lower VAS score and higher WOMAC score compared with the other two groups. For patients with different KOA grades, patients with higher KOA grades were associated with lower grade of treatment efficacy. Conclusions: Patients with KOA, especially those with lower KOA stages, could gain beneficial efficacies from EA treatments with two, four and six points, respectively.
\end{abstract}

\section{Introduction}

As one of the most prevailing forms of osteoarthritis, knee osteoarthritis (KOA) often occurs in the elderly who experience clinical manifestations including pain, inflexibility after getting up or break, ache in the evening, restricted arthrosis movement and arthrosis abnormality [1-3]. KOA is a common, chronic disease which affects one's routine activities L. Qi and Y. Tang contributed equally to this work. 
such as climbing stairs, walking in a short distance and even housekeeping activities [4]. Approximately $60 \%$ males and $70 \%$ females aged above 65 suffer from KOA in the US [5]. The etiological mechanism of KOA can be specified as the followings: it begins with mechanical hindrance in articular cartilage, resilience and sleekness degree were gradually developed and eventually results in reactive bone reshaping, bone spurs, light catagma, dentin degeneration of cartilage and even false cyst [6].

KOA is not only a widespread disease but also irreversible and no curative treatment has been developed. Knee replacement surgery is the most effective approach for managing KOA but this approach is risky for the elderly and the majority of KOA patients are reluctant to have knee replacement surgery [7]. As suggested by the guidelines of the American College of Rheumatology, two effective approaches for alleviating pain resulted from KOA have been discovered including non-pharmacological modalities and pharmacological therapy [8]. Conventional anti-inflammatory drugs are used to alleviate pain of KOA but they have several side-effects such as bleeding and perforated ulcers [9]. Other medicines such as Cyclooxygenase- 2 inhibitors are associated with reduced side effects on gastrointestinal tissues but an increased risk of heart disease [10]. As a result, a large number of drugs have been ruled out since they were not appropriate for managing KOA and a growing number of patients seek help for complementary and alternative therapies that are able to manage KOA in an effective and safe manner [11].

Acupuncture which is originated from ancient China has been widely practiced for thousands of years and it is able to remedy various illnesses with significant efficacy as well as alleviated discomfort [12]. Electroacupuncture (EA) is considered as an improved approach of acupuncture which adds small crocodile clips into the end of acupuncture treatment needles after acupuncture points were connected with an electro-acupuncture device which provided continuous electric stimulations [13]. EA is also considered as a secure and powerful tool for repelling pain $[14,15]$. To date, few studies have confirmed the feasibility of EA in managing KOA patients with respect to pain relief and joint function development [16]. The potential mechanism may be explained by the fact that stimulation of EA induces pro-inflammatory cytokine such as interleukin-6 (IL-6) which may produce anti-inflammatory effects on peripheral receptors $[17,18]$.

Although studies on EA with respect to managing KOA have been reported, few of them are able to interpret the relationship between the number of EA points and therapeutic effects of EA [19]. Taechaarpornkul et al. conducted a comparison between 2-point and 6-point EA in each lap in order to preliminarily investigate whether the number of points significantly affects the efficacy of EA [20]. Moreover, the response of advanced KOA to the same treatment appeared to be less desirable than that of lower-level KOA [21]. Hence, it could be suspected that efficacy exerted by distinct points of EA on KOA could vary with severity of KOA, thereby necessitating a study to profoundly describe how the efficacy of EA of diverse points is related to different stages of KOA.

We carried out a retrospective study in which two-point, four-point and six-point EA was applied to the same number of KOA patients in order to unveil the connection between the number of EA points and its efficacy in managing KOA. The excellent effective rate of treatment in each group were evaluated and compared. Besides that, the Western Ontario and McMaster Universities Osteoarthritis Index (WOMAC) along with the visual analogue scale (VAS) were adopted for pain evaluation and they were used to estimate whether these indexes of treatment efficacy varied among patients with different KOA stages.

\section{Materials and Methods}

Ethics statement

The whole study was agreed by the Institutional Ethics Committee of 181st Central Hospital of The Chinese People's Liberation Army. Informed consents were obtained from patients prior to the inclusion step. 


\section{Cellular Physiology Cell Physiol Biochem 2016;39:2331-2340 \begin{tabular}{l|l|l} 
and Biochemistry $\begin{array}{l}\text { DOI: 10.1159/000447925 } \\
\text { Published online: November 11, } 2016\end{array}$ & $\begin{array}{l}\text { (c) } 2016 \text { The Author(s). Published by S. Karger AG, Basel } \\
\text { www.karger.com/cpb }\end{array}$
\end{tabular} \\ Qi et al.: Eectroacupuncture with Different Points for Knee OA}

Sample size

The minimal sample sizes of three groups (i.e. two-point group, four-point group and six-point group) were estimated with application of PASS 11 before this prospective study (Hintze, J. PASS 11. NCSS, LLC. 2011 Kaysville, Utah, USA. www.ncss.com.). The minimal size was calculated as 37 per group when means/ standard deviations of changed WOMAC scores (after-treatment score-before-treatment score) were set as $19 / 11,21 / 12$ and 11/14, respectively, according to the preliminary clinical tests. Simultaneously, power (1beta) and alpha (significance level) were set as 0.90 and 0.05 , respectively. Considering the possible dropouts and the merits of a big sample size, we initially included 147 KOA patients and 15 subjects dropped out halfway.

\section{Patients and acupuncturists}

A total of 132 KOA patients (30 males and 102 females) were recruited from 181st Central Hospital of The Chinese People's Liberation Army between March 2014 and March 2015. All KOA patients were incorporated according to selection criteria of American College of Rheumatology were then further confirmed by X-ray films [22]. The radiographic assessment was in accordance with Kellgren Lawrence grading scale [23], and patients were accordingly classified into: (1) Grade I: mild osteophyte; (2) Grade II: obvious osteophyte with narrowing joint space; (3) Grade III: moderate narrowing of joint space and sclerosis of subchondral bone; (4) Grade IV: massive osteophyte with obvious narrowing of joint space, as well as severe sclerosis and evident mal-formation of subchondral bone. Patients were excluded from the experiment when: (1) they were uncomfortable with electroacupuncture (EA); (2) their information was missing or incomplete; (3) they had severe cardiac, cerebral, hepatic and renal diseases; (4) they had other inflammatory arthritis that may affect knee functions; (5) they were in the process of pregnancy or lactation; (6) they suffered from mental disease.

The patients were all treated by licensed acupuncturists who were well trained in traditional Chinese medicine with at least three-year clinical experiences. The communication style between acupuncturists and patients were mainly adopted in the form of high expectation. Optimism and hope were conveyed with positive statements, including "We before have proved that this treatment would work", "We have found physical recovery in light of your inspection report" and so on. Besides, the atmosphere on inpatients was maintained as ease and relaxed.

\section{Treatments}

All patients were randomly allocated into three groups including the six-point EA group, four-point EA group and two-point EA group. The randomization was dependent on matched pair design, which was characterized by shortening of individual differences in terms of non-experimental factors, including age, sex ratio, BMI, grading of knees and so on. Patients in the six-point EA group received treatment at six EA points including ST34, SP10, SP9, ST36, ST35 and EX-LE4. Patients in the four-point EA group received treatment at four EA points including ST34, SP10, ST35 and EX-LE5. Finally, patients in the two-point EA group received treatment at only two EA points including ST35 and EX-LE4. Selection of EA points was based on Traditional Chinese Medicine theory which provided reference for local points in order to manage pain areas [20].

Patients were positioned in bed where knees were supported and they remained in a comfortable position for 30 minutes. All patients received EA treatment twice a week for a period of 5 weeks. After disinfecting the local area, sterile needles (30-gauge with $0.32 \mathrm{~mm}$ outer diameter and 50mm length, Suzhou, China) were inserted into EA points with different depths (15-30mm for both ST36 and SP10, 15-25 mm for both SP9 and ST34, 18-25mm for both EX-LE4 and ST35). Electric stimulation was delivered by the medical EA apparatus (G9805, China) for 30 minutes at $0.5 \mathrm{~ms}$ pulse width and $2 \mathrm{~Hz}$ frequency. ST34 was connected to SP10, SP9 was connected to ST36 and ST35 was connected to EX-LE4. Stimulation intensity was continuously adjusted based on patient responses in order to keep the pain level below the threshold.

All treatment groups were advised to have quadriceps exercise for 30 times each day over the experiment period. Apart from that, patients were asked not to have any other therapies including acupuncture treatment, pain-killers or other physical therapies.

Outcome assessments

Treatment outcomes were assessed by Visual Analog Scale (VAS) and McMaster Universities Osteoarthritis Index (WOMAC). VAS is an international scale which reflects pain intensity and it has a 10- 


\section{Cellular Physiology Cell Physiol Biochem 2016;39:2331-2340 \begin{tabular}{l|l|l} 
and Biochemistry & DOI: 10.1159/000447925 & $\begin{array}{l}\text { C } 2016 \text { The Author(s). Published by S. Karger AG, Basel } \\
\text { www.karger.com/cpb }\end{array}$
\end{tabular} \\ Qi et al.: Eectroacupuncture with Different Points for Knee OA}

$\mathrm{cm}$ line ranging from $0 \mathrm{~cm}$ (no pain at all) to $10 \mathrm{~cm}$ (extremely painful) [24]. VAS scores were measured before and after EA treatment for all patients. WOMAC is used to evaluate both pain intensity and functional disability of KOA patients and it adopts a multidimensional measurement of pain, stiffness and disability of physical functions. Five questions were concerned for pain dimension when patients were active or at rest. Two questions were designed for assessing stiffness dimension and seventeen questions were concerned for disability of physical functions [25]. All questions in WOMAC were rated in a numerical scale ranging from 1 (no symptoms) to 5 (severe symptoms). WOMAC was carried out using a self-assessment approach and doctors may provide assistance for those patients who had difficulty in WOMAC assessment. Total scores of the 24 questions were collected, calculated and recorded. A decrease of at least $40 \%$ in patients' WOMAC index score was considered to be statistically significant in treatment efficacy. Regarding patients' VAS, a decrease of at least 30\% compared with baseline was considered as statistically significant in treatment efficacy, and the treatment was believed as extremely effective when the percentage exceeded $60 \%$. The power of the test based on changed WOMAC score was calculated to be $>0.99$. Therapeutic efficacy was evaluated by a self-assessment questionnaire based on "Guiding principle of clinical research on new drugs of traditional Chinese medicine" [26]. A total of 11 items were scored and treatment efficacy was graded into 4 levels. Grade 1 (excellent): symptoms were completely disappeared, joint exhibited normal functions with 0-1 KOA severity scores; grade 2 (good): symptoms mainly disappeared and the majority of joint functions recovered, more than $2 / 3$ of KOA severity scores were reduced and daily activities were unaffected; grade 3 (moderate): pain mainly disappeared, joint extension and flexion exhibited normal functions, some improvement in daily work and activities, less than $2 / 3$ and more than $1 / 3$ KOA severity scores were reduced; grade 4 (bad): less symptoms disappeared and more than $1 / 3$ KOA severity scores were reduced. The total effective rate was defined as the number of knees in grade 1, 2 and 3 out of the total number of knees.

\section{Statistical analysis}

All statistical analyses were performed with SPSS 18.0 software (Chicago, Illinois, USA). Data were presented in the form of mean \pm standard deviation (SD). The two-tailed student's t-test, one-way analysis of variance (ANOVA) or non-parametric test was used to analyze inter-group comparisons of continuous variables, whereas the chi-square test was used for investigating differences in categorical variables between two groups. $\mathrm{P}<0.05$ provided evidence for statistical significance.

\section{Results}

\section{Baseline characteristics of KOA patients}

The clinical characteristics of all patients in the three treatment groups were shown in Table 1. Balanced distribution of clinical characteristics were successfully achieved since there were no significant differences in clinical characteristics including age, sex, pain duration and disease grade among the three treatment groups (all $P>0.05$, Table 1 ).

\section{Comparison of clinical effectiveness among different treatment groups}

The self-assessment conducted on patients with different treatments along with the non-parametric test suggested that no significant difference in the effectiveness of EA was observed among the three treatment groups $(P=0.426)$. The two-point EA group exhibited somewhat lower excellent and effective rate compared with the four-point and six-point EA group (Table 2, Fig. 1).

VAS scores of patients in the three treatment groups exhibited insignificant difference before treatment was applied to patients (all $P>0.05$, Table 3, Fig. 2A). After treatment was applied to patients, the six-point EA group had remarkably lower VAS score compared to the other two groups $(P<0.05)$. By contrast, difference in VAS scores between the two-point and four-point EA group appeared to be insignificant (all $P>0.05$, Table 3, Fig. 2A). Moreover, significant reduction in mean VAS score was observed between pre-treatment VAS and posttreatment VAS in all of the three treatment groups (all $P<0.05$, Table 3, Fig. 2A). 
Table 1. Baseline characteristics of knee OA patients. OA: osteoarthritis. ${ }^{a}$ one-way ANOVA. ${ }^{b}$ chi-square test. SD, standard deviation

\begin{tabular}{lllll}
\hline & $\begin{array}{l}\text { Two-point group } \\
(\mathrm{n}=44)\end{array}$ & $\begin{array}{l}\text { Four-point group } \\
(\mathrm{n}=44)\end{array}$ & $\begin{array}{l}\text { Six-point group } \\
(\mathrm{n}=44)\end{array}$ & $P$ \\
\hline Age, mean \pm SD, years & $59.41 \pm 7.82$ & $61.47 \pm 8.41$ & $60.47 \pm 8.30$ & $0.50^{\mathrm{a}}$ \\
Sex, $\mathrm{n}$ & & & & $1.00^{\mathrm{b}}$ \\
$\quad$ Male & 10 & 10 & 10 & \\
$\quad$ female & 34 & 34 & 34 & \\
$\quad \begin{array}{l}\text { Duration of knee pain, mean } \pm \text { SD, months } \\
\text { Grade, } \mathrm{n}\end{array}$ & $21.84 \pm 9.96$ & $22.23 \pm 10.59$ & $23.25 \pm 10.64$ & $0.81^{\mathrm{a}}$ \\
$\quad \mathrm{I}$ & 10 & 11 & & $0.99^{\mathrm{b}}$ \\
II & 12 & 11 & 9 & \\
$\quad$ III & 12 & 10 & 11 & \\
$\quad$ IV & 10 & 12 & 13 & \\
Target knee, $\mathrm{n}$ & & & & \\
$\quad$ Single knee & 11 & 8 & 10 & \\
$\quad$ Both knees & 33 & 36 & 34 & \\
\hline
\end{tabular}

Table 2. Self-assessment of change in the groups with different treatments. ${ }^{b}$ chi-square test

\begin{tabular}{lllllllll}
\hline Group & $\mathrm{n}$ & Excellent & Good & Moderate & Bad & Excellent rate & Effective rate & $P$ \\
\hline Two-point group & 44 & 8 & 14 & 13 & 9 & 0.18 & 0.80 & \\
Four-point group & 44 & 11 & 16 & $12 /$ & 5 & 0.25 & 0.89 & $0.426^{\text {b }}$ \\
Six-point group & 44 & 10 & 11 & 17 & 6 & 0.23 & 0.86 & \\
\hline
\end{tabular}

A

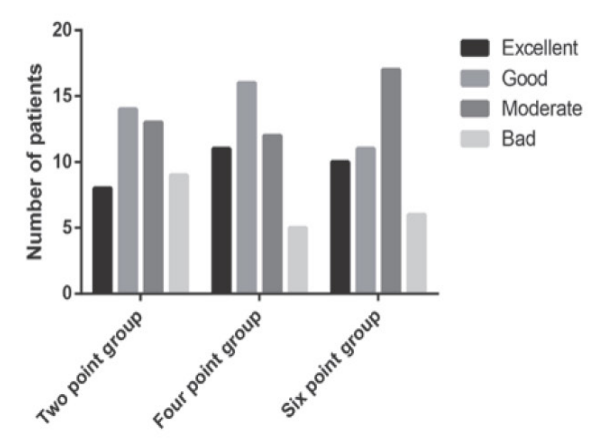

B

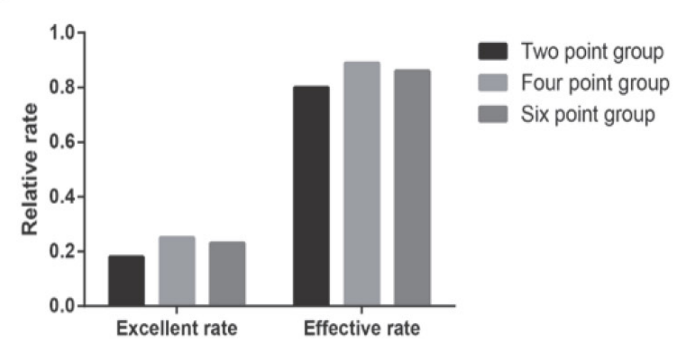

Fig. 1. Self-assessment scores of different treatment groups. A: The efficacy of treatments was graded into different levels including excellent, good, moderate and bad. B: Excellent and effective rate in each treatment group.

On the other hand, the four-point EA group exhibited significantly higher pre-treatment WOMAC scores compared with the six-point EA group $(P<0.05$, Table 3, Fig. $2 \mathrm{~B})$. In addition, the six-point EA group had significantly higher post-treatment WOMAC scores compared to the two-point and four-point EA group (all $P<0.05$, Table 3, Fig. 2B). When we compared pretreatment and post-treatment WOMAC scores, there was significant reduction in WOMAC scores in all of the three treatment groups and therefore we concluded that all of the three EA treatments were significantly effective in managing KOA patients (all $P<0.05$, Table 3, Fig. 2B). 
Table 3. Comparison of VAS and WOMAC scores at baseline and end of treatment between groups with different treatments. VAS: Visual Analog Scale; WOMAC: McMaster Universities Osteoarthritis Index

\begin{tabular}{lclllll}
\hline \multirow{2}{*}{ Group } & \multirow{2}{*}{ VAS } & \multicolumn{2}{c}{ WOMAC } \\
& & Pre-treatment & After-treatment & $p$ & Pre-treatment & After-treatment
\end{tabular}

Table 4. Self-assessment of change in the groups with different grades of knee OA. ${ }^{\mathrm{b}}$ chi-square test

\begin{tabular}{lllllllll}
\hline Group & $\mathrm{n}$ & Excellent & Good & Moderate & Bad & Excellent rate & Effective rate & $P$ \\
\hline Grade I & 30 & 11 & 10 & 8 & 1 & 0.37 & 0.97 & \\
Grade II & 34 & 9 & 8 & 11 & 5 & 0.26 & 0.85 & $0.005^{\mathrm{b}}$ \\
Grade III & 33 & 8 & 10 & 9 & 6 & 0.24 & 0.82 & \\
Grade IV & 35 & 1 & 11 & 15 & 8 & 0.03 & 0.77 & \\
\hline
\end{tabular}

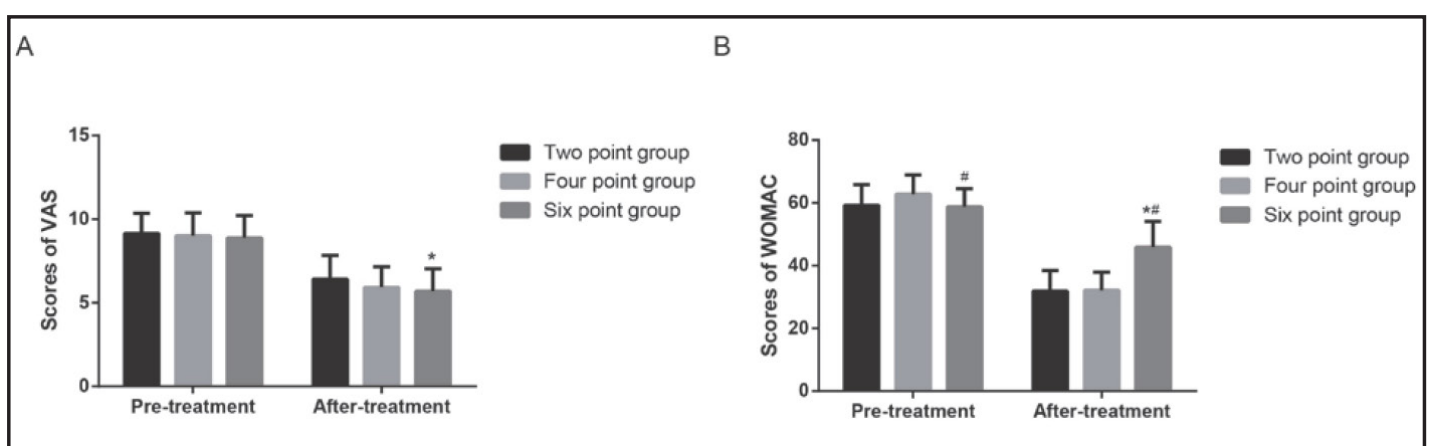

Fig. 2. Pre-treatment and post-treatment VAS (A) and WOMAC (B) scores in different treatment groups. Values were shown as mean \pm SD. ${ }^{*}$ denotes significant differences. VAS: Visual Analog Scale; WOMAC: McMaster Universities Osteoarthritis Index; SD: standard deviation.

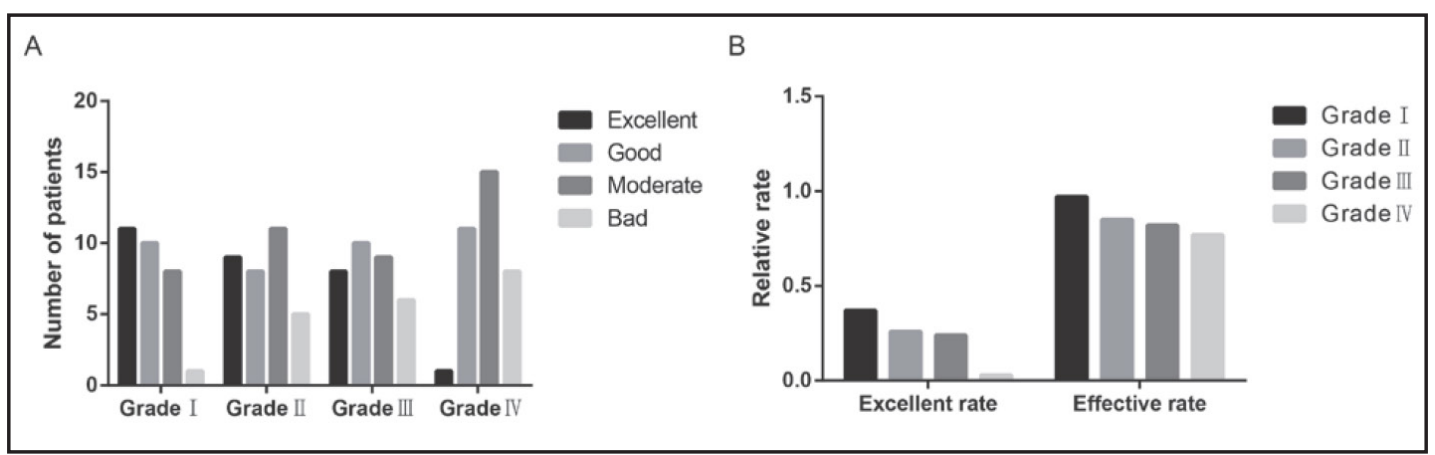

Fig. 3. Self-assessment scores of different KOA grades. A: The efficacy of treatments was ranked into different levels including excellent, good, moderate and bad. B: Excellent and effective rate of each treatment group. OA: osteoarthritis.

\section{Comparing clinical effectiveness of EA for KOA patients with different grades}

Results from self-assessment of KOA patients indicated that significant differences in the effectiveness of EA were detected among the Grade I, II, III and IV group $(P=0.005$, Table 4, Fig. 3A). The Grade I group exhibited significantly more excellent and effective rate whereas the Grade IV group exhibited the least excellent and effective rate (Table 4, Fig. 3B). 
Table 5. Comparison of VAS and WOMAC scores at baseline and end of treatment between groups with different grades of knee $\mathrm{OA}$

\begin{tabular}{|c|c|c|c|c|c|c|c|}
\hline \multirow{2}{*}{ Group } & \multirow{2}{*}{$\mathrm{n}$} & \multicolumn{2}{|l|}{ VAS } & \multicolumn{3}{|c|}{ WOMAC } & \multirow[b]{2}{*}{$P$} \\
\hline & & Pre-treatment & After-treatment & $P$ & Pre-treatment & After-treatment & \\
\hline Grade I & 30 & $8.87 \pm 0.35$ & $5.63 \pm 0.49$ & & $59.47 \pm 2.18$ & $34.67 \pm 6.36$ & \\
\hline Grade II & 34 & $8.97 \pm 0.17$ & $5.85 \pm 0.56$ & & $60.00 \pm 1.92$ & $35.88 \pm 6.89$ & \\
\hline Grade III & 33 & $9.03 \pm 0.17$ & $5.97 \pm 0.68$ & & $60.27 \pm 1.94$ & $36.79 \pm 6.75$ & \\
\hline Grade IV & 35 & $9.28 \pm 0.45$ & $6.34 \pm 0.94$ & & $61.21 \pm 1.99$ & $40.07 \pm 6.91$ & \\
\hline
\end{tabular}

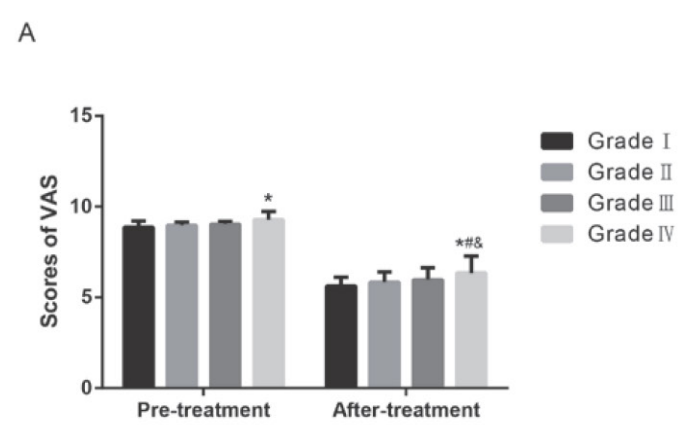

B

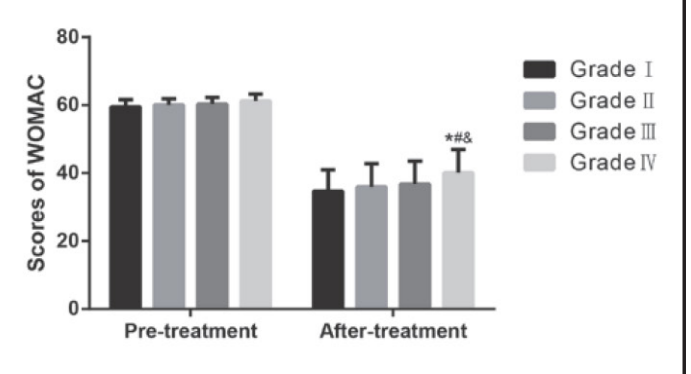

Fig. 4. Pre-treatment and post-treatment VAS (A) and WOMAC (B) scores in different KOA grades. Values were shown as mean $\pm \mathrm{SD}$. ${ }^{*}$ denotes significant differences.

More importantly, we discovered that patients with higher grades of KOA were associated with less effectiveness of EA. Therefore, we also concluded that electroacupuncture may be less effective for patients with higher grades of KOA.

We also compared both pre-treatment and post-treatment VAS scores for KOA patients with different grades and the Grade IV group exhibited significantly higher pre-treatment VAS scores compared to the Grade I group $(P<0.05$, Table 5, Fig. $4 A)$. The Grade IV group had significantly higher post-treatment VAS scores in comparison to the Grade I, II and III group (all $P<0.05$ ), while such significant difference was not presented among the Grade I, II and III group (all $P>0.05$, Table 5, Fig. 4A). Besides that, significant reduction in mean VAS score between the pre-treatment and post-treatment VAS scores was observed in the Grade I, II, III and IV group (all $P<0.05$, Table 5, Fig. 4A). There was no significant difference in pre-treatment WOMAC among the four groups of patients with different grades (all $P>0.05$, Table 5, Fig. 4B). The Grade IV group exhibited significantly higher post-treatment WOMAC scores compared with the Grade I, II and III group (all $P<0.05$ ), while such difference among the Grade I, II and III group was not significant (all $P>0.05$, Table 5, Fig. 4B). There was significant reduction in the average WOMAC scores after EA treatments were applied to KOA patients and this trend was consistent across different groups of KOA grades (all $P<0.05$, Table 5, Fig. 4B).

\section{Discussion}

The present study was designed to compare the efficacy of electroacupuncture among $132 \mathrm{KOA}$ patients who were allocated to three groups of EA with different number of EA points. Each group was separately treated with two-point, four-point or six-point EA and the effectiveness of EA were assessed by VAS and WOMAC scales. As suggested by both VAS and WOMAC scores, all of the three EA treatments were therapeutically effective for managing KOA patients, whereas the self-assessment scale suggested no significant difference in the effectiveness of EA among the three treatment groups. Since VAS score indicates the pain 
intensity around the knee joint and WOMAC score indicates the severity of KOA symptoms, then significant reduction in VAC and WOMAC scores provided evidence for the effectiveness of EA in managing KOA patients. Results from our study were consistent with those from other randomized controlled trials (RCT) which concluded significant efficacy of EA in managing KOA patients. For example, Taechaarpornkul et al. discovered that the average WOMAC scores of both two-point and six-point EA were significantly reduced over a fiveweek treatment period [20]. Apart from that, Jubb et al. also acknowledged that manual acupuncture combined with EA could significantly alleviate KOA-related symptoms compared to the sham acupuncture group [27]; Witt et al. also discovered that improvement in joint function and pain of KOA patients was more significant in the standard EA treatment group compared with the minimal or no acupuncture treatment group [28]. However, other researchers may argue that EA was not significantly effective in managing KOA patients. For instance, Suarez-Almazor et al. conducted a RCT over a period of 3 month and concluded that the EA treatment group was not superior over the sham acupuncture group in managing KOA patients [29]. A few factors may influence the effectiveness of EA in managing KOA patients and these factors include patient expectation, operation temperature, number of needles, electric stimulation intensity and treatment duration [30]. In addition, some authors suggested that acupuncturist may have substantial impact on the effectiveness of EA treatment $[29,31]$.

Furthermore, varied stimulation conditions such as the number of points and treatment intensity are likely to have some impact on the effectiveness of EA. Our study demonstrated that the effective rate in the two-point group was slightly lower than those of the other two groups and this may imply that EA with more stimulated points are more appropriate for managing KOA patients. A large number of studies suggested that strong EA stimulations were associated with the optimal treatment effectiveness of EA [28]. For example, Vas employed all local EA points except ST 36 and achieved the highest tolerable intensity [32] whereas Ahsin concluded that two-point EA (ST35, EX-LE4) was more appropriate than sixpoint EA $[13,20]$. As a result of this, more evidence should be obtained in order to verify the optimal number of EA points.

Our study also provided evidence that the effectiveness of EA significantly varied among patients at different stages. The effective rate of Grade IV group was 0.77 which was significantly lower than those of the other three groups. Self-assessment scores, VAS scores and WOMAC scores all indicated that EA was significantly effective in managing KOA patients. Apart from that, EA was more appropriate for mild and moderate KOA patients than for severe KOA patients.

Several limitations of our study should also be acknowledged. Firstly, confounding factors like patients' expectations and acupuncturists' bias couldn't be totally avoided as well. Secondly, we have only adopted VAS, WOMAC and the self-assessment questionnaire of patients as assessments of EA effectiveness. Other indexes including Timed Up-and-Go Test (TUGT) score [19], Lequesne's functional index and 50 feet-walk time [33] may be considered as alternative. Finally, the follow-up period was only 1 year, and long-term changes might arguably be more clinically relevant to patients with KOA.

In conclusion, EA with different acupuncture points may not exert different effectiveness in KOA patients, but it has different roles in KOA patients at different stages. Patients with KOA, especially those with lower KOA grades could gain short-term benefits from EA treatments with all the two-, four- and six- points, implying that EA with those points could effectively and alleviate the clinical symptoms among KOA patients.

\section{Acknowledgements}

This study was supported by The research and development program of health suitable technology for Guangxi (S201407-03, and The scientific research and technological development project of Guilin (20140120-1-8).

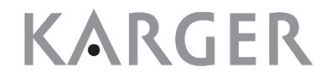




\section{Cellular Physiology Cell Physiol Biochem 2016;39:2331-2340 and Biochemistry \begin{tabular}{l|l} 
DOI: 10.1159/000447925 & (c) 2016 The Author(s). Published by S. Karger AG, Basel \\
www.karger.com/cpb
\end{tabular} \\ Qi et al.: Eectroacupuncture with Different Points for Knee OA}

\section{Disclosure Statement}

The authors declare no commercial or financial conflict of interest.

\section{References}

1 Uth K, Trifonov D: Stem cell application for osteoarthritis in the knee joint: A minireview. World J Stem Cells 2014;6:629-636.

2 Altman RD, Hochberg MC: Degenerative joint disease. Clin Rheum Dis 1983;9:681-693.

3 Lemperg RK, Arnoldi CC: The significance of intraosseous pressure in normal and diseased states with special reference to the intraosseous engorgement-pain syndrome. Clin Orthop Relat Res 1978;143-156.

4 Guccione AA, Felson DT, Anderson JJ, Anthony JM, Zhang Y, Wilson PW, Kelly-Hayes M, Wolf PA, Kreger BE, Kannel WB: The effects of specific medical conditions on the functional limitations of elders in the Framingham Study. Am J Public Health 1994;84:351-358.

5 Sarzi-Puttini P, Cimmino MA, Scarpa R, Caporali R, Parazzini F, Zaninelli A, Atzeni F, Canesi B: Osteoarthritis: an overview of the disease and its treatment strategies. Semin Arthritis Rheum 2005;35:1-10.

6 Setnikar I: Antireactive properties of "chondroprotective" drugs. Int J Tissue React 1992;14:253-261.

7 Katz JN: Total joint replacement in osteoarthritis. Best Pract Res Clin Rheumatol 2006;20:145-153.

8 Recommendations for the medical management of osteoarthritis of the hip and knee: 2000 update. American College of Rheumatology Subcommittee on Osteoarthritis Guidelines. Arthritis Rheum 2000;43:1905-1915.

9 Tramer MR, Moore RA, Reynolds DJ, McQuay HJ: Quantitative estimation of rare adverse events which follow a biological progression: a new model applied to chronic NSAID use. Pain 2000;85:169-182.

10 Juni P, Reichenbach S, Egger M: COX 2 inhibitors, traditional NSAIDs, and the heart. BMJ 2005;330:13421343.

11 Cao XW, Guo D, Liu JW, Niu W, Liu J, Pan JK, Xie H, Ouyang WW, Lin DK: The efficacy and safety of the Shaoyao Shujin tablet for knee osteoarthritis: study protocol for a randomized controlled trial. Trials 2016;17:3.

12 Li A, Zhang Y, Lao L, Xin J, Ren K, Berman BM, Zhang RX: Serotonin Receptor 2A/C Is Involved in Electroacupuncture Inhibition of Pain in an Osteoarthritis Rat Model. Evid Based Complement Alternat Med 2011;2011:619650.

13 Ahsin S, Saleem S, Bhatti AM, Iles RK, Aslam M: Clinical and endocrinological changes after electroacupuncture treatment in patients with osteoarthritis of the knee. Pain 2009;147:60-66.

14 He LF: Involvement of endogenous opioid peptides in acupuncture analgesia. Pain 1987;31:99-121.

15 Gordon JS: Alternative medicine and the family physician. Am Fam Physician 1996;54:2205-2212, 2218 2220.

16 Eisenberg DM, Davis RB, Ettner SL, Appel S, Wilkey S, Van Rompay M, Kessler RC: Trends in alternative medicine use in the United States, 1990-1997: results of a follow-up national survey. JAMA 1998;280:15691575.

17 Liu X, Shen L, Wu M, Wu B, Gao L, Hu W, Zhang A: Effects of acupuncture on myelogenic osteoclastogenesis and IL-6 mRNA expression. J Tradit Chin Med 2004;24:144-148.

18 Chen XH, Han JS: Analgesia induced by electroacupuncture of different frequencies is mediated by different types of opioid receptors: another cross-tolerance study. Behav Brain Res 1992;47:143-149.

19 Ng MM, Leung MC, Poon DM: The effects of electro-acupuncture and transcutaneous electrical nerve stimulation on patients with painful osteoarthritic knees: a randomized controlled trial with follow-up evaluation. J Altern Complement Med 2003;9:641-649.

20 Taechaarpornkul W, Suvapan D, Theppanom C, Chanthipwaree C, Chirawatkul A: Comparison of the effectiveness of six and two acupuncture point regimens in osteoarthritis of the knee: a randomised trial. Acupunct Med 2009;27:3-8.

21 Knoop J, Dekker J, van der Leeden M, van der Esch M, Klein JP, Hunter DJ, Roorda LD, Steultjens MP, Lems WF: Is the severity of knee osteoarthritis on magnetic resonance imaging associated with outcome of exercise therapy? Arthritis Care Res (Hoboken) 2014;66:63-68. 


\section{Cellular Physiology Cell Physiol Biochem 2016;39:2331-2340 \begin{tabular}{ll|l} 
DOI: 10.1159/000447925 & $\begin{array}{l}\text { O 2016 The Author(s). Published by S. Karger AG, Basel } \\
\text { www.karger.com/cpb }\end{array}$
\end{tabular} \\ Qi et al.: Eectroacupuncture with Different Points for Knee OA}

22 Altman R, Asch E, Bloch D, Bole G, Borenstein D, Brandt K, Christy W, Cooke TD, Greenwald R, Hochberg M: Development of criteria for the classification and reporting of osteoarthritis. Classification of osteoarthritis of the knee. Diagnostic and Therapeutic Criteria Committee of the American Rheumatism Association. Arthritis Rheum 1986;29:1039-1049.

23 Kellgren JH, Lawrence JS: Radiological assessment of osteo-arthrosis. Ann Rheum Dis 1957;16:494-502.

24 Jensen MP, Karoly P, Braver S: The measurement of clinical pain intensity: a comparison of six methods. Pain 1986;27:117-126.

25 Bellamy N, Buchanan WW, Goldsmith CH, Campbell J, Stitt LW: Validation study of WOMAC: a health status instrument for measuring clinically important patient relevant outcomes to antirheumatic drug therapy in patients with osteoarthritis of the hip or knee. J Rheumatol 1988;15:1833-1840.

26 Wu MX, Li XH, Lin MN, Jia XR, Mu R, Wan WR, Chen RH, Chen LH, Lin WQ, Huang CY, Zhang XR, Hong KD, Li L, Liu XX: Clinical study on the treatment of knee osteoarthritis of Shen-Sui insufficiency syndrome type by electroacupuncture. Chin J Integr Med 2010;16:291-297.

27 Jubb RW, Tukmachi ES, Jones PW, Dempsey E, Waterhouse L, Brailsford S: A blinded randomised trial of acupuncture (manual and electroacupuncture) compared with a non-penetrating sham for the symptoms of osteoarthritis of the knee. Acupunct Med 2008;26:69-78.

28 Witt C, Brinkhaus B, Jena S, Linde K, Streng A, Wagenpfeil S, Hummelsberger J, Walther HU, Melchart D, Willich SN: Acupuncture in patients with osteoarthritis of the knee: a randomised trial. Lancet 2005;366:136-143.

29 Suarez-Almazor ME, Looney C, Liu Y, Cox V, Pietz K, Marcus DM, Street RL, Jr.: A randomized controlled trial of acupuncture for osteoarthritis of the knee: effects of patient-provider communication. Arthritis Care Res (Hoboken) 2010;62:1229-1236.

30 Vas J, White A: Evidence from RCTs on optimal acupuncture treatment for knee osteoarthritis--an exploratory review. Acupunct Med 2007;25:29-35.

31 Liu T: Role of acupuncturists in acupuncture treatment. Evid Based Complement Alternat Med 2007;4:3-6.

32 Vas J, Mendez C, Perea-Milla E, Vega E, Panadero MD, Leon JM, Borge MA, Gaspar O, Sanchez-Rodriguez F, Aguilar I, Jurado R: Acupuncture as a complementary therapy to the pharmacological treatment of osteoarthritis of the knee: randomised controlled trial. BMJ 2004;329:1216.

33 Sangdee C, Teekachunhatean S, Sananpanich K, Sugandhavesa N, Chiewchantanakit S, Pojchamarnwiputh S, Jayasvasti S: Electroacupuncture versus diclofenac in symptomatic treatment of osteoarthritis of the knee: a randomized controlled trial. BMC Complement Altern Med 2002;2:3. 